ToKyo J. MATH.

VoL. 19, No. 1, 1996

\title{
On Helicoidal Surfaces with Constant Mean Curvature and Their Limiting Surfaces
}

\author{
Takao SASAI \\ Tokyo Metropolitan University \\ Dedicated to Professor Kenjiro Okubo on his 60th birthday
}

\section{Introduction.}

In this paper we shall study the family of helicoidal surfaces $f: M^{2} \rightarrow \mathbf{E}^{3}$ with non-zero constant mean curvature in the Euclidean 3-space $\mathbf{E}^{3}$, which was investigated by M. P. doCarmo and M. Dajczer [doC-D]. They obtained an integral representation of $f$ which contains three parameters, say $B, h$ and $m$ (see Theorem 1.1 below). It gives a natural generalization of a representation for Delaunay surfaces, i.e., the surfaces of revolution with constant mean curvature, due to $\mathrm{K}$. Kenmotsu [K].

Furthermore, for a given helicoidal surface $f: M^{2} \rightarrow \mathbf{E}^{3}$, they determined a $2 \pi$ periodic parametrization of a family of immersions $f_{\theta}: M^{2} \rightarrow \mathbf{E}^{3}, \theta \in[0,2 \pi], f_{0}=f$, all of which are isometric to $f$ and have the same constant mean curvature $H$ (see Theorem 1.3). The family $\left\{f_{\theta}\right\}$ is called the associated family to $f$ and contains, except for a few cases, just two different Delaunay surfaces. Lawson's theorem ([L], see also [doC-D], p. 426) says that the family $\left\{f_{\theta}\right\}$ contains all local isometric immersions with given $H$. In $§ 1$ we shall summarize several known results for the later use.

Their representation of the family $\left\{f_{\theta}\right\}$ emphasizes the Delaunay surfaces as the starting point of deformations. However, for an arbitrarily given helicoidal surface, it is slightly hard to find the family to which it belongs. So, in $\S 2$, we shall construct a new parametrization $S(B, \mathfrak{h}, \mathfrak{m})$ of the family which also depends on one parameter $B$. It takes a given helicoidal surface as the starting point of its deformations from which the corresponding values of the remaining parameters $h$ and $m$ are calculated at once. If a given helicoidal surface $f$ is not the right circular cylinder, then, in the both of these parametrizations $f_{\theta}$ and $S(B, \mathfrak{h}, \mathfrak{m})$, the parameter $B$ moves on the closed interval bounded by $B^{+}$and $1 / B^{+}$(see (A. 1) in $\S 2$ ), which is determined uniquely by $f$. However, it seems unnatural that, in the family $\left\{f_{\theta}\right\}$ for the right circular cylinder corresponding to $B=0$, the parameter $B$ stays at zero only. Moreover, there appears only one limiting

Received August 1, 1994 
helicoidal surface which corresponds to $B=0$ and $h, m \rightarrow+\infty$ in this family, but there exist infinitely many limiting helicoidal surfaces. In $\S 3$ we shall study the family of such surfaces which are the deformations of the right circular cylinder by virtue of asymptotic behaviours of the integral representations. Moreover, in $\$ 4$, we shall determine all limiting helicoidal surfaces which constitute, we may say, the boundary of the family of all helicoidal surfaces with constant mean curvature $H$.

The author wishes to thank Professor K. Voss and Professor K. Ogiue who read manuscript carefully and gave various kind advice.

\section{Basic facts.}

Let $(x, y, z)$ be coordinates in $\mathbf{E}^{3}$. We consider the one-parameter subgroup $\left\{g_{t}\right.$; $-\infty<t<\infty\}$ of the group of rigid motions of $\mathbf{E}^{3}$ given by

$$
g_{t}(x, y, z)=(x \cos t-y \sin t, x \sin t+y \cos t, z+h t),
$$

where $-\infty<h<+\infty$ is a constant. The motion $g_{t}$ is called a helicoidal motion with axis the $z$-axis and pitch $h$. A helicoidal surface with axis the z-axis and pitch $h$ is a surface which is invariant under $g_{t}$ for all $t$. If $h \neq 0$, the orbit $\left\{g_{t}(x, y, z) ; t \in \mathbf{R}\right\}$ of a point not lying on the $z$-axis is a circular helix with axis the $z$-axis and that of a point on the $z$-axis is the whole $z$-axis. When $h=0$, the motions reduce to rotations about the $z$-axis and generate a surface which is called a surface of revolution or a rotation surface.

We particularly note the cases where $h \rightarrow \pm \infty$. In these cases the motions reduce to translations and the orbits degenerate to straight lines parallel to the $z$-axis. Then the motions generate cylinders as limiting helicoidal surfaces (for details, see (B2) in §3).

Now we identify a helicoidal surface with an immersion $f: M^{2} \rightarrow \mathbf{E}^{3}$ of a domain $M^{2}$ with coordinates $(s, t)$. The so-called natural parametrization of a helicoidal surface (limiting or not) is characterized as follows (see [doC-D], pp. 426-427): $t$-curves, i.e., the images by $f$ of the curves given by $s=$ constant, are the orbits of helicoidal motions, while $s$ is the arclength of the curves orthogonal to those orbits. We note that the first fundamental form in these parameters is written as

$$
d \sigma^{2}=d s^{2}+U^{2}(s) d t^{2}, \quad U(s)>0 .
$$

On the other hand, on an open set $D \subset M$, we may assume that the intersection of $f(D)$ with some plane $\pi \subset \mathbf{E}^{3}$ containing the $z$-axis is a graph $z=\lambda(\rho)$ over the intersection of $\pi$ with the $x y$-plane. Then the restriction $\left.f\right|_{D}$ can be represented by

$$
f(\rho, \varphi)=(\rho \cos \varphi, \rho \sin \varphi, \lambda(\rho)+h \varphi),
$$

where $(\rho, \varphi)$ are the polar coordinates in the $x y$-plane. Moreover we easily obtain that $U / \sqrt{\rho^{2}+h^{2}}$ is non-zero constant. Then, by setting $m=\sqrt{\rho^{2}+h^{2}} / U$ and integrating (1), we see that the natural parametrization of the helicoidal surface (2) with given first fundamental form (1) can be calculated by 
(3)

$$
\left\{\begin{array}{l}
\rho=\sqrt{m^{2} U^{2}-h^{2}} \\
\varphi=\frac{t}{m}-\frac{h}{m} \int \frac{\sqrt{m^{2} U^{2}\left(1-m^{2} \dot{U}^{2}\right)-h^{2}}}{U\left(m^{2} U^{2}-h^{2}\right)} d s \\
\lambda=m \int \frac{U \sqrt{m^{2} U^{2}\left(1-m^{2} \dot{U}^{2}\right)-h^{2}}}{m^{2} U^{2}-h^{2}} d s
\end{array}\right.
$$

where the dot denotes the differentiation with respect to $s$ ([doC-D], pp, 428-429). In (3) the (trivial) integral constants are already eliminated by a rigid motion of the coordinate axis and by adjusting the origin of the parameter $t$. Thus, for any helicoidal surface, there is a two-parameter family of helicoidal surfaces isometric to it, which is essentially parametrized by $m$ and $h$; this is just Bour's lemma.

From (1) and (3) we easily obtain

$$
\begin{gathered}
\frac{1}{m^{2}} \geq \dot{U}^{2}, \quad U^{2} \geq U^{2}\left(1-m^{2} \dot{U}^{2}\right) \geq \frac{h^{2}}{m^{2}} \geq 0, \\
\boldsymbol{H}=\frac{1-m^{2} \dot{U}^{2}-m^{2} U \ddot{U}}{2 \sqrt{m^{2} U^{2}\left(1-m^{2} \dot{U}^{2}\right)-h^{2}}},
\end{gathered}
$$

where $\boldsymbol{H}$ and $\boldsymbol{K}$ are the mean and Gaussian curvatures of the surface, respectively.

From now on we only consider the case where the mean curvature $\boldsymbol{H}$ is non-zero constant $H$. Then, in this case, the differential equation (5) can easily be solved ([doCD], p. 430). By introducing a new parameter B given by

$$
U^{2}=\frac{1+B^{2}+4 H^{2} h^{2}+2 B \sin 2 H s}{4 m^{2} H^{2}},
$$

we obtain:

THEOREM 1.1 (doCarmo-Dajczer). The helicoidal surface (2) given by (3) has constant mean curvature $H \neq 0$ if and only if

$$
\left\{\begin{array}{l}
\rho=\frac{\sqrt{1+B^{2}+2 B \sin 2 H s}}{2 H}, \\
\varphi=\frac{t}{m}-4 H^{2} h \int \frac{1+B \sin 2 H s}{\left(1+B^{2}+2 B \sin 2 H s\right) \sqrt{1+B^{2}+4 H^{2} h^{2}+2 B \sin 2 H s}} d s \\
\lambda=\int \frac{(1+B \sin 2 H s) \sqrt{1+B^{2}+4 H^{2} h^{2}+2 B \sin 2 H s}}{1+B^{2}+2 B \sin 2 H s} d s .
\end{array}\right.
$$


Moreover it gives a natural parametrization of the surface.

REMARK 1.2. When the parameters $B$ and $h$ are fixed in (8), the various surfaces given by different finite values of $m$ have the same image with distinct parametrization. However, the parameter $m$ plays an important role in constructing the associated family to a given surface.

Let us denote by $U(B, h, m)$ the representation of $U$ given in (7) and, by $S(B, h, m)$ the surface corresponding to it. On the other hand, for $-\infty<B_{0}<+\infty$ and $0<\left|m_{0}\right|<$ $+\infty$, we set

$$
\left\{\begin{array}{l}
B=B\left(B_{0}, \theta\right)=\frac{2 B_{0}}{2+\left(B_{0}^{2}-1\right)(1-\cos \theta)}, \\
h=h\left(B_{0}, \theta\right)=\frac{\left(B_{0}^{2}-1\right) \sin \theta}{2 H\left[2+\left(B_{0}^{2}-1\right)(1-\cos \theta)\right]} \\
m^{2}=m^{2}\left(B_{0}, m_{0}, \theta\right)=\frac{2 m_{0}^{2}}{2+\left(B_{0}^{2}-1\right)(1-\cos \theta)},
\end{array}\right.
$$

and we define a helicoidal surface $f\left(B_{0}, m_{0} ; \theta\right)=S\left(B\left(B_{0}, \theta\right), h\left(B_{0}, \theta\right), m\left(B_{0}, m_{0}, \theta\right)\right)$. Then $f\left(B_{0}, m_{0} ; \theta+2 \pi\right)=f\left(B_{0}, \mathrm{~m}_{0} ; \theta\right)$ is obvious, i.e., this parametrization with parameter $\theta$ is $2 \pi$-periodic.

TheOREM 1.3 (doCarmo-Dajczer). (1) For any Delaunay surface $S\left(B_{0}, 0, m_{0}\right)$ $\left(-\infty<B_{0}<+\infty, 0<\left|m_{0}\right|<+\infty\right),(B, h, m)$ given by (9) satisfy

$$
U\left(B\left(B_{0}, \theta\right), h\left(B_{0}, \theta\right), m\left(B_{0}, m_{0}, \theta\right)\right)=U\left(B_{0}, 0, m_{0}\right)
$$

for all $-\infty<\theta<+\infty$, and then, $\left\{f\left(B_{0}, m_{0} ; \theta\right) \mid 0 \leq \theta \leq 2 \pi\right\}$ is the associated family to $f\left(B_{0}, m_{0} ; 0\right)=S\left(B_{0}, 0, m_{0}\right)$.

(2) For any helicoidal surface $S(B, h, m)(-\infty<B, h<+\infty, 0<|m|<+\infty)$ with constant mean curvature $H \neq 0$, there exist $\left(B_{0}, m_{0}, \eta\right)$ satisfying

$$
B=B\left(B_{0}, \eta\right), \quad h=h\left(B_{0}, \eta\right), \quad m=m\left(B_{0}, m_{0}, \eta\right)
$$

and, consequently, the family $\left\{f\left(B_{0}, m_{0} ; \theta\right)\right\}$ given by those $B_{0}, m_{0}$ and $(9)$ is the associated family to $S(B, h, m)$ and, in particular, to the Delaunay surface $S\left(B_{0}, 0, m_{0}\right)$.

REMARK 1.4. The parametrization (9) obviously emphasizes the Delaunay surface $S\left(B_{0}, 0, m_{0}\right)$ as the starting point of the deformation and, if $B_{0} \neq 0, \pm 1$, it determines just one more Delaunay surface $S\left(1 / B_{0}, 0, \mathrm{~m}_{0} / B_{0}\right)=f\left(B_{0}, m_{0} ; \pi\right)$; one of them is an unduloid and the other is a nodoid.

REMARK 1.5. From now on we may assume $H>0$ without loss of generality. As we know already, for any $B_{0} \neq 0, \pm 1, B$ in (9) varies monotonically from $B_{0}$ to $1 / B_{0}$ on $[0, \pi]$ and return to $B_{0}$ on $[\pi, 2 \pi]$. Then we expect that, if $B_{0}=0, B$ varies from 0 
to $\pm \infty$ but, to the contrary, it stays at $B=0$ alone. Furthermore we have to notice that, in all families $\left\{f\left(B_{0}, m_{0} ; \theta\right) \mid 0 \leq \theta \leq 2 \pi\right\}$ given by (9) under the conditions $-\infty<$ $B_{0}<+\infty$ and $0<\left|m_{0}\right|<+\infty$, only $B_{0}=0$ is the case which contains a limiting helicoidal surface $S(0, \pm \infty, \pm \infty)=f\left(0, m_{0} ; \pi\right)$.

Finally we define limiting helicoidal surfaces in general for the later sections.

Definition 1.6. The surface $S(B, h, m)$ defined by (2) and (8) is called a limiting helicoidal surface if at least one of the quantities $|B|,|h|$ and $|m|$ takes the value $+\infty$.

\section{Isometric deformations when $B_{0} \neq 0$.}

Besides the condition that $H$ is a positive constant, we also assume that $-\infty<B$, $h<+\infty$ and $0<|m|<+\infty$. Let us fix such constants $B_{0}, h_{0}$ and $m_{0}$. Then, by (3) and (8), they determine the helicoidal surfaces $S\left(B_{0}, h_{0}, m_{0}\right)$ with constant mean curvature $H$. Let $S(B, h, m)$ be another helicoidal surface with the same $H$. We parametrize those by the common coordinates $(s, t)$ given in (8). Then, from (7), $S(B, h, m)$ is isometric to $S\left(B_{0}, h_{0}, m_{0}\right)$ if and only if the following relations hold;

$$
\frac{1+B^{2}+4 H^{2} h^{2}}{m^{2}}=\frac{1+B_{0}^{2}+4 H^{2} h_{0}^{2}}{m_{0}^{2}}, \quad \frac{B}{m^{2}}=\frac{B_{0}}{m_{0}^{2}} .
$$

In this section we consider the case $B_{0} \neq 0$ only.

(A.1) At first, set $\Delta=1+B_{0}^{2}+4 H^{2} h_{0}^{2}$ and, further, $B^{ \pm}\left(B_{0}, h_{0}\right)=\left(\Delta \pm \sqrt{\Delta^{2}-4 B_{0}^{2}}\right) /$ $\left(2 B_{0}\right)$. Thus, for given $B_{0}>0$ (resp. $\left.B_{0}<0\right)$ and $-\infty<h_{0}<+\infty, B^{-}\left(B_{0}, h_{0}\right) \leq B^{+}\left(B_{0}, h_{0}\right)$ (resp. $B^{-} \geq B^{+}$) are uniquely determined, where " $=$" holds if and only if both $B_{0}=1$ (resp. $\left.B_{0}=-1\right)$ and $h_{0}=0$. An elementary calculation also shows that

$$
B^{-}\left(B_{0}, h_{0}\right) \cdot B^{+}\left(B_{0}, h_{0}\right)=1 .
$$

(A.2) Eliminating the parameter $m$ in the first relation in (10) by using the second one, we easily obtain

$$
h^{2}=\left(1 / 4 H^{2}\right)\left[B^{+}\left(B_{0}, h_{0}\right)-B\right]\left[B-B^{-}\left(B_{0}, h_{0}\right)\right] .
$$

Thus $B$ must be in the closed interval $I=\left[B^{-}\left(B_{0}, h_{0}\right), B^{+}\left(B_{0}, h_{0}\right)\right]$ (resp. $=\left[B^{+}, B^{-}\right]$). Let us denote by $\mathfrak{h}\left(B_{0}, h_{0} ; B\right)$ the positive branch of $h$ on $I$ determined by (11). Then, $\mathfrak{h}\left(B_{0}, h_{0} ; B_{0}\right)=h_{0}$ or $-h_{0}$ according as $h_{0}>0$ or $<0$ and, obviously, $\mathfrak{h}\left(B_{0}, h_{0} ; B\right)=0$ if and only if either $B=B^{+}\left(B_{0}, h_{0}\right)$ or $B^{-}\left(B_{0}, h_{0}\right)$. When $B_{0}=1$ (resp. $\left.=-1\right)$ and $h_{0}=0$, $I$ is reduced to one point $\{B=1\}$ (resp. $\{B=-1\}$ ).

(A.3) We define two mappings $\varphi^{+}$and $\varphi^{-}: \boldsymbol{I} \rightarrow \boldsymbol{I} \times \mathbf{R}$ by $\varphi^{ \pm}(B)=\left(B, \pm \mathfrak{h}\left(B_{0}, h_{0}\right.\right.$; $B)$ ). Let us denote the open interval $\left(B^{-}\left(B_{0}, h_{0}\right), B^{+}\left(B_{0}, h_{0}\right)\right)\left(\operatorname{resp} .\left(B^{+}, B^{-}\right)\right)$by $I^{i}$. Then we easily obtain: 
LEMMA 2.1. (1) On the closed interval $I, \varphi^{ \pm}: I \rightarrow I \times \mathbf{R}$ are continuous, one-to-one mappings onto $\varphi^{ \pm}(I)$, respectively, and are analytic on $I^{i}$.

(2) $B^{ \pm}\left(B_{0}, h_{0}\right)=B^{ \pm}\left(B, \mathfrak{h}\left(B_{0}, h_{0} ; B\right)\right)=B^{ \pm}\left(B,-\mathfrak{h}\left(B_{0}, h_{0} ; B\right)\right)$ for all $B \in I$, i.e., $B^{ \pm}$'s are invariant on $\varphi^{+}(I)$ and $\varphi^{-}(I)$. Consequently, for any $\left(B_{1}, h_{1}\right) \in \varphi^{ \pm}(I), \mathfrak{h}\left(B_{0}, h_{0} ; B\right)=$ $\mathfrak{h}\left(B_{1}, h_{1} ; B\right)$ holds.

(A.4) Since $B B_{0}>0$ for all $B \in I$ and $m_{0} \neq 0$, we may choose $m$, from the second relation in (10), as $m=m_{0}\left(B / B_{0}\right)^{1 / 2}$, and denote it by $m\left(B_{0}, m_{0} ; B\right)$. The assumption $B_{0} \neq 0$ implies $B^{-}\left(B_{0}, h_{0}\right) \neq 0$ and, thus, $m\left(B_{0}, m_{0} ; B\right) \neq 0$ is an analytic function of $B$ on I. From Lemma 2.1 we obtain.

Proposition 2.2. A surface $S(B, h, m)$ is isometric to $S\left(B_{0}, h_{0}, m_{0}\right)$ if and only if the following two conditions hold;

(1) $B^{ \pm}(B, h)=B^{ \pm}\left(B_{0}, h_{0}\right)$,

(2) $m=m_{0}\left(B / B_{0}\right)^{1 / 2}$.

(A.5) Corresponding to a given helicoidal surface $S\left(B_{0}, h_{0}, m_{0}\right)$, we define two families of isometric helicoidal surfaces with given $H$ as

$$
\begin{aligned}
& \mathscr{D}^{+}=\left\{S\left(B, \mathfrak{h}\left(B_{0}, h_{0} ; B\right), \mathfrak{m}\left(B_{0}, m_{0} ; B\right)\right) \mid B \in I\right\}, \\
& \mathscr{D}^{-}=\left\{S\left(B,-\mathfrak{h}\left(B_{0}, h_{0} ; B\right), \mathfrak{m}\left(B_{0}, m_{0} ; B\right)\right) \mid B \in I\right\},
\end{aligned}
$$

and set $\mathscr{D}=\mathscr{D}^{+} \cup \mathscr{D}^{-}$. Then $\mathscr{D}$ is a one-parameter family of helicoidal surfaces depending continuously on B and differentiable on $I^{i}$ from Lemma 2.1. The given surface $S\left(B_{0}, h_{0}, m_{0}\right)$ belongs to $\mathscr{D}^{+}$or $\mathscr{D}^{-}$according as $h_{0}>0$ or $<0$, and $\mathscr{D}^{+} \cap \mathscr{D}^{-}$consists of, in general, two different Delaunay surfaces $S\left(B^{ \pm}\left(B_{0}, h_{0}\right), 0, m\left(B_{0}, m_{0} ; B^{ \pm}\left(B_{0}, h_{0}\right)\right)\right)$.

(A.6) Now we recall the associated family stated in $\$ 1$. For a given helicoidal surface $S\left(B_{0}, h_{0}, m_{0}\right), B\left(B^{-}\left(B_{0}, h_{0}\right), \theta\right)$ (resp. $\left.B\left(B^{+}\left(B_{0}, h_{0}\right), \theta\right)\right)$ varies monotonically from $B^{-}\left(B_{0}, h_{0}\right)$ (resp. $\left.B^{+}\left(B_{0}, h_{0}\right)\right)$ to $B^{+}\left(B_{0}, h_{0}\right)$ (resp. $\left.B^{-}\left(B_{0}, h_{0}\right)\right)$ on $[0, \pi]$ and returns monotonically to $B^{-}\left(B_{0}, h_{0}\right)$ (resp. $\left.B^{+}\left(B_{0}, h_{0}\right)\right)$ on $[\pi, 2 \pi]$. Thus $B\left(B^{ \pm}\left(B_{0}, h_{0}\right),[0, \pi]\right)=$ $B\left(B^{ \pm}\left(B_{0}, h_{0}\right),[\pi, 2 \pi]\right)=I$. For brevity we simply denote $B\left(B^{ \pm}\left(B_{0}, h_{0}\right), \theta\right), h\left(B^{ \pm}\left(B_{0}, h_{0}\right), \theta\right)$ and $m\left(B^{ \pm}\left(B_{0}, h_{0}\right), m\left(B_{0}, m_{0} ; B^{ \pm}\left(B_{0}, h_{0}\right)\right), \theta\right)$ by $B^{ \pm}(\theta), h^{ \pm}(\theta)$ and $m^{ \pm}(\theta)$, respectively. Then we have

$$
\begin{gathered}
h^{-}(\theta)=\left\{\begin{array}{rrr}
-\mathfrak{h}\left(B_{0}, h_{0} ; B^{-}(\theta)\right) & \text { on } & {[0, \pi],} \\
\mathfrak{h}\left(B_{0}, h_{0} ; B^{-}(\theta)\right) & \text { on } & {[\pi, 2 \pi],}
\end{array}\right. \\
h^{+}(\theta)=\left\{\begin{array}{rrr}
\mathfrak{h}\left(B_{0}, h_{0} ; B^{+}(\theta)\right) & \text { on } & {[0, \pi],} \\
-\mathfrak{h}\left(B_{0}, h_{0} ; B^{+}(\theta)\right) & \text { on } & {[\pi, 2 \pi],}
\end{array}\right. \\
m^{ \pm}(\theta)=\mathfrak{m}\left(B_{0}, m_{0} ; B^{ \pm}(\theta)\right) .
\end{gathered}
$$

These relations and Proposition 2.2 lead the following. 
Proposition 2.3.

$$
\begin{aligned}
\mathscr{D} & =\left\{f\left(B^{ \pm}(0), m^{ \pm}(0) ; \theta\right) \mid 0 \leq \theta \leq 2 \pi\right\} \\
& =\left\{f\left(B^{ \pm}\left(B_{0}, h_{0}\right), \mathfrak{m}\left(B_{0}, m_{0} ; B^{ \pm}\left(B_{0}, h_{0}\right)\right) ; \theta\right) \mid 0 \leq \theta \leq 2 \pi\right\} .
\end{aligned}
$$

REMARK 2.4. We note that $d B / d \theta=0$ for $\theta \equiv 0$ (mod. $\pi$ ). This is the reason why the analyticity in $\mathscr{D}$ breaks down at $B=B^{ \pm}\left(B_{0}, h_{0}\right)$.

REMARK 2.5. For given $S\left(B_{0}, h_{0}, m_{0}\right)$, there exists another family of helicoidal surfaces isometric to it. It is obtained by replacing $m\left(B_{0}, m_{0} ; B\right)$ by $-\mathfrak{m}\left(B_{0}, m_{0} ; B\right)$ in $\mathscr{D}$. In other words the one more family is led from $\mathscr{D}$ by changing the parameter $t$ into $-t$.

\section{Isometric deformations when $B_{0}=0$.}

In the following argument we may assume, by the respective changes of $s$ and $t$ into $-s$ and $-t$ if necessary, that $B \geq 0$ and $m>0$. Moreover we may assume, without loss of generality, that $B_{0}=0$ and $h_{0}=0$, that is, $S\left(B_{0}, h_{0}, m_{0}\right)$ is a right circular cylinder as a surface of revolution with $d \sigma^{2}=d s^{2}+d t^{2} /\left(4 m_{0}^{2} H^{2}\right), K=0$ and $\boldsymbol{H}=H$. The relations (10) are rewritten as

$$
\frac{1+B^{2}+4 H^{2} h^{2}}{m^{2}}=\frac{1}{m_{0}^{2}}, \quad \frac{B}{m^{2}}=0 .
$$

We consider all cases that preserve these relations. They can be classified into four cases:

(B1) $B=0,0<h, m \leq+\infty$,

(B2) $0<B<+\infty, h, m=+\infty$,

(B3) $B=+\infty, 0 \leq h<+\infty, m=+\infty$,

(B4) $B, h, m=+\infty$.

Now we recall the definition of the asymptotic equivalence:

DEFINITION 3.1. Let $F(\tau)$ and $G(\tau)$ be positive valued functions of $\tau \geq 0$. They are said to be asymptotically equivalent for large $\tau$ and denoted by $F(\tau) \approx G(\tau)$ if

(a) $F(\tau) / G(\tau) \rightarrow 1$ for $\tau \rightarrow+\infty$, when $F(\tau), G(\tau) \rightarrow 0$ for $\tau \rightarrow+\infty$,

(b) $|F(\tau)-G(\tau)| \rightarrow 0$ for $\tau \rightarrow+\infty$, when $F(\tau), G(\tau) \rightarrow+\infty$ for $\tau \rightarrow+\infty$.

(B1) This case is already given by (9). In fact, setting $B_{0}=0$ in (9), we obtain $m^{2}=m_{0}^{2}\left(1+4 H^{2} h^{2}\right)$ for $0<h, m<+\infty$ and $m^{2} \approx 4 H^{2} m_{0}^{2} h^{2}$ for $h \rightarrow+\infty$. Thus the first relation in (12) is satisfied and the second is obvious from the conditions on (B1). As is already known ([doC-D], see also $[\mathrm{H}-\mathrm{R}])$, the surfaces $S(0, h, m)(0<h, m<+\infty)$ and $S(0,+\infty,+\infty)$ are also the right circular cylinders as helicoidal and translational surfaces, respectively (for the latter case, see the next paragraph).

(B2) From (12) we again obtain $m^{2} \approx 4 m_{0}^{2} H^{2} h^{2}$ for sufficiently large $h \gg 1$. Then, by (8), the relations 


$$
\left\{\begin{array}{l}
\rho=\frac{\sqrt{1+B^{2}+2 B \sin 2 H s}}{2 H}, \\
\varphi \approx \frac{t}{2 m_{0} H h}-2 H \int \frac{1+B \sin 2 H s}{1+B^{2}+2 B \sin 2 H s} d s, \\
\lambda \approx 2 H h \int \frac{1+B \sin 2 H s}{1+B^{2}+2 B \sin 2 H s} d s,
\end{array}\right.
$$

follow and, consequently, $\lambda+h \varphi \approx t /\left(2 m_{0} H\right)$. We denote by $I$ the integral of the righthand-side in (13) and set

$$
\Phi(B, s)=\operatorname{Arcsin} \frac{2 B+\left(1+B^{2}\right) \sin 2 H s}{1+B^{2}+2 B \sin 2 H s},
$$

where Arcsin denotes the principal value of the arcsine function. Then $I$ is given by

$$
I= \begin{cases}\frac{1}{2}[s \pm(1 / 2 H) \Phi(B, s)], & \text { for }-\pi /(4 H) \leq s \leq \pi /(4 H), \\ \frac{1}{2}[s \pm(1 / 2 H)\{\pi-\Phi(B, s)\}], & \text { for } \pi /(4 H) \leq s \leq 3 \pi /(4 H), \\ \frac{1}{2}[s \pm(1 / 2 H)\{2 \pi+\Phi(B, s)\}], & \text { for } 3 \pi /(4 H) \leq s \leq 5 \pi /(4 H) \\ \ldots \ldots \ldots \ldots \ldots \ldots \ldots, & \end{cases}
$$

where \pm takes + or - according as $0 \leq B<1$ or $B>1$. We also obtain $I=s / 2$ for $B=1$. Then, for $B \neq 1$ and any fixed $t$, the curve determined by (2) and (13) is a circle in the plane $\left\{z=t /\left(2 m_{0} H\right)\right\}$. Thus we conclude that, for $h \rightarrow+\infty$, the surface tends to a right circular cylinder parallel to the $z$-axis.

The surface $S(B,+\infty,+\infty)$ is actually given by

$$
\left\{\begin{array}{l}
\rho=\frac{\sqrt{1+B^{2}+2 B \sin 2 H s}}{2 H}, \\
\varphi=-2 H \int \frac{1+B \sin 2 H s}{1+B^{2}+2 B \sin 2 H s} d s, \\
z=\lambda+h \varphi=t /\left(2 m_{0} H\right)
\end{array}\right.
$$

which is a right circular cylinder with constant mean curvature $H$. Its intersection with the $x y$-plane is the circle $x^{2}+(y+B /(2 H))^{2}=1 /\left(4 H^{2}\right)$ or $(x-B /(2 H))^{2}+y^{2}=1 /\left(4 H^{2}\right)$ according as $0 \leq B<1$ or $1<B<+\infty$.

When $B=1, \varphi$ is reduced to $-H s$ and the surface $S(1,+\infty,+\infty)$ is given by

$$
x=\frac{\sqrt{2+2 \sin 2 H s}}{2 H} \cos H s, \quad y=-\frac{\sqrt{2+2 \sin 2 H s}}{2 H} \sin H s, \quad z=\frac{t}{2 m_{0} H} .
$$

It looks like a couple of the same circular cylinders which are tangent along the $z$-axis 
HELICOIDAL SURFACES

47

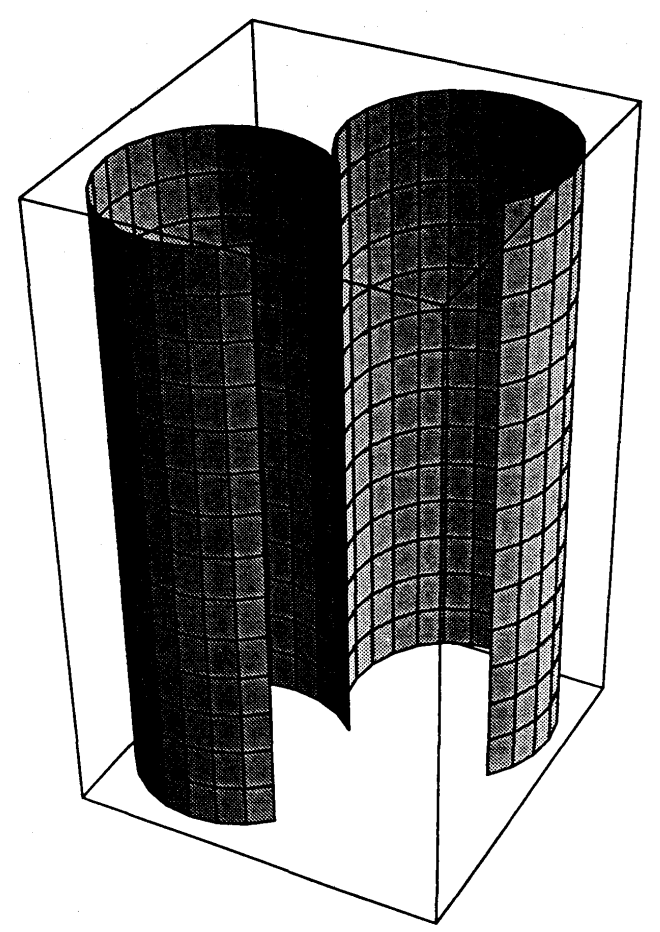

FIGURE 1. $B=1, h, m \rightarrow+\infty, 0 \leq s \leq(3 / 2) \pi$, $\Delta s=\pi / 24$

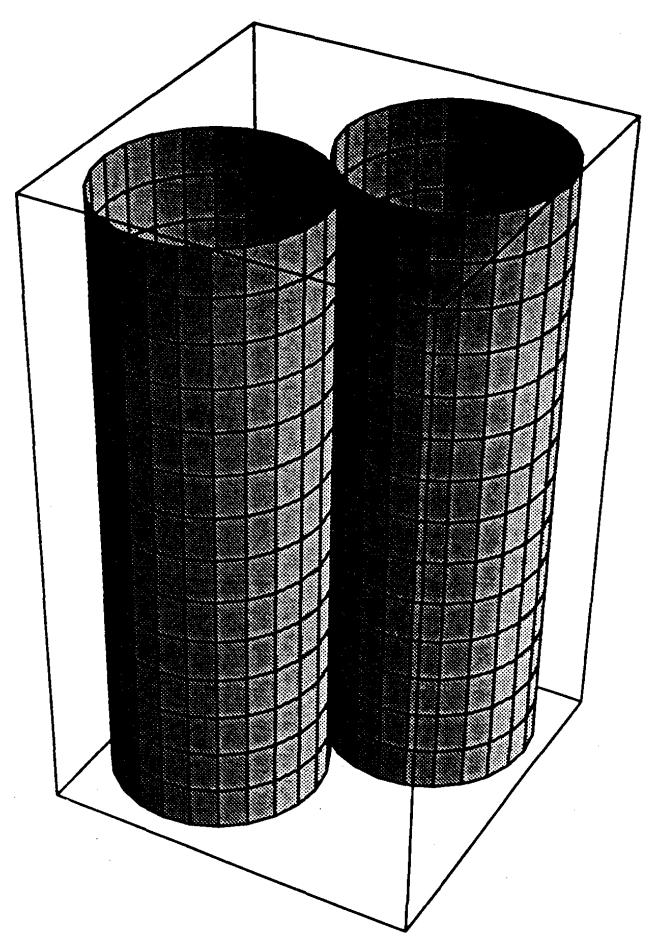

Figure 2. $B=1, h, m \rightarrow+\infty, 0 \leq s \leq 2 \pi$, $\Delta s=\pi / 24$

(see Figures 1 and 2). More explicitly, its intersection with the $x y$-plane is a reducible curve given by $2 H^{2}\left(x^{2}+y^{2}\right)-(x-y)^{2}=0$ whose two irreducible components are tangent at $x=y=0$. The surface $S(1,+\infty,+\infty)$ is certainly isometric to $S\left(0,0, m_{0}\right)$.

Proposition 3.2. For any $0 \leq B<+\infty$, the surface $S(B,+\infty,+\infty)$ defined by (2) and (14) is a circular cylinder which is isometric to $S\left(0,0, m_{0}\right)$ and has constant mean curvature $H$. The family $\{S(B,+\infty,+\infty) \mid 0 \leq B<+\infty\}$ depends analytically on $B$ in the interval $[0,1)$ and $(1,+\infty)$, and is discontinuous at $B=1$.

REMARK 3.3. In case $B \neq 1$ above, the centers of the circles lie on the $x$ - or $y$-axis. It is caused by eliminating an integral constant in (3). In fact, if we replace $\varphi$ by $\varphi+\alpha$ $(\alpha=$ constant $)$ in (8), those centers lie on the line $y=(\tan \alpha) x$ or $y=-x /(\tan \alpha)$.

(B3) From (12) we have $m^{2} \approx m_{0}^{2} B^{2}(B \rightarrow+\infty)$ and, consequently,

$$
\left\{\begin{array}{l}
\rho \approx \frac{1}{2 H}(B+\sin 2 H s) \\
\varphi \approx \frac{t}{m_{0} B}-\frac{4 H^{2} h}{B^{2}} \int \sin 2 H s d s \approx \frac{t}{m_{0} B}, \\
\lambda \approx \int \sin 2 H s d s=-\frac{1}{2 H} \cos 2 H s
\end{array}\right.
$$


Thus $\lambda+h \varphi \approx-(1 / 2 H) \cos 2 H s+h\left(t / m_{0} B\right)$. For any $0 \leq B<+\infty$, the surface $S[B, h]$ defined by (2) and (15) is a helicoidal surface with axis the $z$-axis and pitch $h$. For example, when $h=0$, it is a surface of revolution whose generating curve is given by $(x-B /(2 H))^{2}+z^{2}=1 /\left(4 H^{2}\right)$, i.e., it is a torus. In terms of the parametrization (15), the first fundamental form $d \sigma^{2}$ and the curvatures $K, H$ of $S[B, h]$ can be calculated by an elementary computation. Furthermore we can easily see that; when $B \rightarrow+\infty$,

$$
d \sigma^{2} \rightarrow d s^{2}+\frac{d t^{2}}{4 m_{0}^{2} H^{2}}, K \rightarrow 0 \text { and } \boldsymbol{H} \rightarrow H, \quad \text { uniformly }
$$

Though the relations (8) are reduced, if $B \rightarrow+\infty$, to $\rho=+\infty, \varphi=0$ and $\lambda=$ $-(1 / 2 H) \cos 2 H s$, but, geometrically, we may naturally comprehend the surface $S(+\infty, h,+\infty)$ as the limit of those images of immersions in $\mathbf{E}^{3}$, e.g., $S(+\infty, 0,+\infty)$ is the torus whose generating curve is given, symbolically, by $(x-(+\infty))^{2}+z^{2}=1 /\left(4 H^{2}\right)$.

Summarizing the above arguments we may assert:

THEOREM 3.4. For any $0<B<+\infty$ and $0 \leq h<+\infty$, the surface $S[B, h]$ determined by (2) and (15) is a helicoidal surface with axis the $z$-axis and pitch $h$. The surface $S(+\infty, h,+\infty)$ is given as the limiting surface of the images of $S[B, h]$ 's in $\mathbf{E}^{3}$. It is a helicoidal surface generated by the circle with center at the point at infinity and radius $1 / 2 H$, which is isometric to $S\left(0,0, m_{0}\right)$ and has constant mean curvature $H$.

REMARK 3.5. If one stands at any point near such a torus or a helicoidal surface, the surface looks like one or infinitely many circular cylinders, respectively. In the latter case they are parallel at equal spaces and, are overlapping or not according to the value of $h$.

(B4) As mentioned above, there is no concrete parametrization for expressing the surface $S(+\infty, h,+\infty)$. Therefore, if we consider the surface $S(+\infty,+\infty,+\infty)$ as the limit of $S(+\infty, h,+\infty)$ 's, we can no longer examine it by studying asymptotic behaviours of those surfaces. However, in such a consideration, a geometric meaning of $S(+\infty,+\infty,+\infty)$ can easily be imagined; $\cdots$ it must be a circular cylinder parallel to the $z$-axis and standing around the point at infinity. And it is true. We certainly obtain this fact by studying the limit of $S(B,+\infty,+\infty)$ 's, $B \rightarrow+\infty$, as follows.

For sufficiently large $B$, we have, from (14),

$$
\rho \approx \frac{1}{2 H}(B+\sin 2 H s), \quad \varphi \approx \frac{1}{B} \cos 2 H s, \quad z=\lambda+h \varphi=\frac{t}{2 m_{0} H} .
$$

We consider the surface $S[B]$ defined by (2) and (16), i.e.,

$$
\left\{\begin{array}{l}
x=(1 / 2 H)(B+\sin 2 H s) \cos [(1 / B) \cos 2 H s], \\
y=(1 / 2 H)(B+\sin 2 H s) \sin [(1 / B) \cos 2 H s], \\
z=t /\left(2 m_{0} H\right) .
\end{array}\right.
$$


If $B$ is sufficiently large, then $(1 / B) \cos 2 H s$ is sufficiently small, and we have

$$
\begin{aligned}
(x-B /(2 H))^{2}+y^{2} & =\frac{1}{2 H^{2}} B(B+\sin 2 H s)\left\{1-\cos \left[\frac{1}{B} \cos 2 H s\right]\right\}+\frac{1}{4 H^{2}} \sin ^{2} 2 H s \\
& \approx \frac{1}{4 H^{2}} B(B+\sin 2 H s)\left(\frac{\cos 2 H s}{B}\right)^{2}+\frac{1}{4 H^{2}} \sin 2 H s \approx \frac{1}{4 H^{2}} .
\end{aligned}
$$

Moreover, as in (B3), the first fundamental form and curvatures, $\boldsymbol{K}$ and $\boldsymbol{H}$, tend respectively to $d s^{2}+d t^{2} /\left(4 m_{0}^{2} H^{2}\right), 0$ and $H$.

Thus we may assert:

THEOREM 3.6. For any $0<B<+\infty$, the surface $S[B]$ defined by (2) and (16) is $a$ cylinder parallel to the $z$-axis. The surface $S(+\infty,+\infty,+\infty)$ is given as the limiting surface of $S[B]$ 's. It is a circular cylinder whose intersection with the $x y$-plane is a circle given by $(x-(+\infty))^{2}+y^{2}=1 /\left(4 H^{2}\right)$. It is also isometric to $S\left(0,0, m_{0}\right)$ and has constant mean curvature $H$.

\section{Limiting helicoidal surfaces.}

Several kinds of limiting helicoidal surfaces were studied in the previous section. We recall the Definition 1.6 and list all possible cases in the following;

\begin{tabular}{c|c|c|c|c|c|c|c} 
case & 1 & 2 & 3 & 4 & 5 & 6 & 7 \\
\hline$|B|$ & $<+\infty$ & $<+\infty$ & $<+\infty$ & $=+\infty$ & $=+\infty$ & $=+\infty$ & $=+\infty$ \\
$|h|$ & $<+\infty$ & $=+\infty$ & $=+\infty$ & $<+\infty$ & $<+\infty$ & $=+\infty$ & $=+\infty$ \\
$|m|$ & $=+\infty$ & $<+\infty$ & $=+\infty$ & $<+\infty$ & $=+\infty$ & $<+\infty$ & $=+\infty$
\end{tabular}.

In all these cases the function $U$ is reduced to a constant. Here we note that it is natural to consider limiting surfaces under the following condition:

(\#) For the limiting helicoidal surface under consideration, there always exists an isometric helicoidal surface $S\left(B_{0}, h_{0}, m_{0}\right)$ with $\left|B_{0}\right|<+\infty,\left|h_{0}\right|<+\infty$ and $0<\left|m_{0}\right|<+\infty$, which has the same constant mean curvature $H \neq 0$.

Then there is no necessity for studying the cases where $U \equiv 0$ or $U \equiv+\infty$. Thus the Cases 1, 2, 4 and 6 are excluded from our consideration.

By the relations (10) and the above condition (\#), we conclude that $B_{0}$ must be zero. Thus we may take $S\left(0,0, m_{0}\right)$ as $S\left(B_{0}, h_{0}, m_{0}\right)$ and, consequently, the Cases 3 and 5 are essentially the same as (B2) and (B3) in $\$ 3$, respectively.

For the Case 7, we already observed $\lim _{B \rightarrow \infty} S(B,+\infty,+\infty)$ and $\lim _{h \rightarrow \infty} S(+\infty$, $h,+\infty)$ in (B4) in $\S 3$. Therefore, from the relations (12), it is sufficient to consider the following three situations: For sufficiently large $m$,

(C7) $h^{2} \approx m^{2} /\left(4 m_{0}^{2} H^{2}\right)$ and $\lim _{m \rightarrow \infty} B / m=0$, 
(C7) $B_{2} B^{2} \approx m^{2} / m_{0}^{2}$ and $\lim _{m \rightarrow \infty} h / m=0$,

(C7) $3 \approx k_{1} m, h \approx k_{2} m\left(k_{1}, k_{2} \neq 0\right)$ and $k_{1}^{2}+4 H^{2} k_{2}^{2}=1 / m_{0}^{2}$.

Obviously the classes $(\mathrm{C} 7)_{1}$ and $(\mathrm{C} 7)_{2}$ respectively contain (B2) and (B3) as special cases. In each case of $(\mathrm{C} 7)_{i}$ 's, we can easily show, by a method similar to that in $\S 3$, that the limiting surface is the right circular cylinder standing around the point at infinity with radius $1 / 2 H$. Namely the result is the same as (B4).

THEOREM 4.1. Under the condition (\#), limiting helicoidal surfaces are those stated in Proposition 3.2, Assertions 3.4 and 3.6. Each surface is a flat surface with given constant mean curvature $H \neq 0$.

\section{References}

[B] E. Bour, Memoire sur le deformation de surfaces, J. École Polytech. 39 (1862), 1-148.

[doC-D] M. P. DoCARmo and M. DAJCZER, Helicoidal surfaces with constant mean curvature, Tôhoku Math. J. 34 (1982), 425-435.

[D] C. Delaunay, Sur la surface de revolution dont la courbure moyenne est constante, J. Math. Pures Appl. Ser. 16 (1841), 309-320.

[H-R] L. R. HitT and I. M. Roussos, Computer graphics of helicoidal surfaces with constant mean curvature, preprint.

[K] K. Kenmotsu, Surfaces of revolution with prescribed mean curvature, Tôhoku Math. J. 32 (1980), 147-153.

[L] H. B. Lawson, JR, Complete minimal surfaces in $S^{3}$, Ann. of Math. 92 (1970), 335-374.

Present Address:

Department of Mathematics, Tokyo Metropolitan University, Minami-OHSAWA, HaChioji-ShI, ToKyo, 192-03 JAPAN. 